University of Nebraska - Lincoln

DigitalCommons@University of Nebraska - Lincoln

Faculty Publications, Department of Physics and Astronomy

Research Papers in Physics and Astronomy

2009

\title{
A wide-angle electron grating bi-prism beam splitter
}

\author{
Adam Caprez \\ University of Nebraska-Lincoln, acaprez2@unl.edu \\ Roger Bach \\ University of Nebraska-Lincoln, roger.bach@gmail.com \\ Scot McGregor \\ University of Nebraska-Lincoln \\ Herman Batelaan \\ University of Nebraska - Lincoln, hbatelaan@unl.edu
}

Follow this and additional works at: https://digitalcommons.unl.edu/physicsfacpub

Part of the Physics Commons

Caprez, Adam; Bach, Roger; McGregor, Scot; and Batelaan, Herman, "A wide-angle electron grating biprism beam splitter" (2009). Faculty Publications, Department of Physics and Astronomy. 93.

https://digitalcommons.unl.edu/physicsfacpub/93

This Article is brought to you for free and open access by the Research Papers in Physics and Astronomy at DigitalCommons@University of Nebraska - Lincoln. It has been accepted for inclusion in Faculty Publications, Department of Physics and Astronomy by an authorized administrator of DigitalCommons@University of Nebraska Lincoln. 


\title{
A wide-angle electron grating bi-prism beam splitter
}

\author{
Adam Caprez ${ }^{1}$, Roger Bach, Scot McGregor and Herman Batelaan \\ Department of Physics and Astronomy, University of Nebraska-Lincoln, Lincoln, NE 68588-0111, \\ USA \\ E-mail: adam.caprez@huskers.unl.edu
}

Received 21 April 2009, in final form 11 June 2009

Published 22 July 2009

Online at stacks.iop.org/JPhysB/42/165503

\begin{abstract}
We demonstrate a wide-angle electron beam splitter capable of producing $1 \mathrm{~cm}$ beam separation at the detection plane. The beam splitter utilizes a nanofabricated periodic grating in combination with a bi-prism element. In contrast to devices utilizing only bi-prism elements, the use of the periodic grating causes amplitude, and not wavefront, splitting. Even at maximum separation, beam profiles remain undistorted, providing evidence that coherence is intact. This is a step towards the realization of a large area electron interferometer using such a grating bi-prism combination.
\end{abstract}

\section{Introduction}

For the past half-century, electron interferometers have been used for both fundamental physics and more applied areas [1]. The shorter de Broglie wavelength of electrons provides electron interferometers with a much finer measuring 'comb' than their optical counterparts. The electron's charge also provides for a strong coupling to its environment. This combination has made electron interferometers a powerful tool for the study of fundamental physics. The first electron interferometer was constructed using metallic crystals as diffractive elements in 1953 [2, 3]. Shortly afterwards, an interferometer using a bi-prism wire in lieu of metallic crystals was demonstrated in 1955 [4]. All subsequent devices fell into these two basic types until recently, when interferometers using nanofabricated gratings were realized in 2006 [5-7].

More recently, applications of large area interferometers have become of interest, spurring the further development of electron matter optics elements. For instance, determining the electron forward-scattering amplitude with atoms or molecules by placing a gas cell in one arm of the interferometer requires large beam separation [8]. Also, the separation distance controls decoherence induced by nearby surfaces and relates to studies of the quantum-classical boundary [9]. A large area electron interferometer may also be the first step towards a proposed novel method of high-sensitivity rotation sensing using a charged particle interferometer enclosed in a Faraday

\footnotetext{
1 Author to whom any correspondence should be addressed.
}

cage [10]. The application, which the authors are pursuing is a test of the dispersionless nature of the Aharonov-Bohm effect [11]. Such a test requires placing a large solenoid between the arms of an interferometer.

As a first step towards this goal, our group has previously demonstrated high-quality electron diffraction from a nanofabricated grating [12]. To ensure that the diffracted beams are also coherent, our group has also previously demonstrated a three-grating Mach-Zehnder interferometer [5]. However, a small separation between the electron beams (3 $\mu \mathrm{m})$ does not allow for objects to be placed between, or in, one of the interferometer arms. In this paper, we report the construction of a large angle beam splitter composed of a nanofabricated grating in conjunction with a bi-prism wire.

A schematic of the experimental setup is shown in figure 1. A Kimball Physics EGG-3101 electron gun was used as a thermionic source at the energy of $7.5 \mathrm{keV}$ with an estimated $\Delta E$ of $1 \mathrm{eV}$. All electron optics elements aside from the electron gun are rigidly mounted on a rail system. Two layers of magnetic shielding inside the vacuum system enclose the rail system. The inside layer is grounded at a single point to minimize eddy currents and thus provide shielding to oscillating magnetic fields. An external Faraday cage provides shielding from stray electric fields. A $2 \mu \mathrm{m}$ diameter molybdenum circular aperture at a distance of $12 \mathrm{~cm}$ from the electron gun provides beam collimation. A second identical aperture, $18 \mathrm{~cm}$ behind the first, further narrows beam divergence. The beam is incident on a $100 \mathrm{~nm}$ periodicity nanofabricated grating situated $7 \mathrm{~cm}$ from the second aperture. 


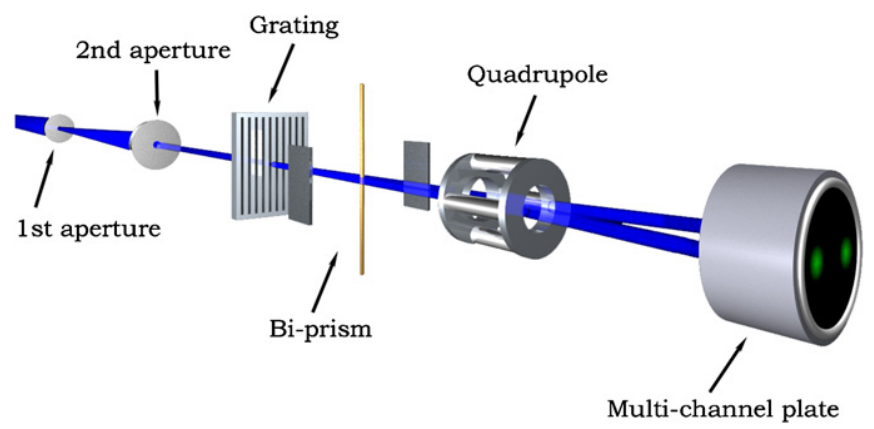

Figure 1. Schematic of experimental setup. Two apertures collimate an electron beam; a grating then coherently splits the beam. The zero-order diffraction beam is blocked by the bi-prism wire, while the dominant first-order beams pass on either side. The wire increases the beam separation without broadening, while the quadrupole magnifies the entire diffraction pattern. A multi-channel plate and a fluorescent screen are used to image the pattern.

The grating used is identical to those used by Gronniger et al [5]. The spatial transverse coherence length of the electron beam incident on the grating is estimated to be $750 \mathrm{~nm}$, based on the ratio of diffraction-order separation to beam width and grating periodicity [12].

As the beam encounters the grating it undergoes diffraction, with the angles at which the maxima occur given by

$$
n \lambda_{\mathrm{dB}}=d \sin \left(\theta_{n}\right),
$$

where $n$ is the order number, $\lambda_{\mathrm{dB}}$ is the de Broglie wavelength of the electrons, $d$ is the grating periodicity and $\theta_{n}$ is the diffraction angle. The quality of the diffraction pattern is good, and similar gratings have produced resolved orders out to the positive and negative 21 st order [12]. In this study, the positive and negative first-order beams are used. The diffracted beam is aligned such that the zeroth order is centred on, and thus mostly blocked, by the bi-prism wire.

The wire is placed at a distance of $5.5 \mathrm{~cm}$ from the grating. The mount for the wire is shown in figure 2. The wire itself is composed of a quartz glass fibre that has been coated with gold via sputtering to a thickness of approximately $100 \mathrm{~nm}$. The quartz fibre is produced by rapidly stretching a rod of quartz that has been melted. A similar technique for wire production is described by Hibi and Yada [13]. The resulting thin thread is then mounted on an electrically insulating ring (composed of Ultem). The diameter of the wire for the data in this work is $5 \mu \mathrm{m}$.

A voltage $V_{\mathrm{bp}}$ applied to the bi-prism wire gives a potential surrounding the wire which can be approximated as [1]

$$
V(r)=V_{\mathrm{bp}} \frac{\ln \left(r / R_{\mathrm{el}}\right)}{\ln \left(R_{\mathrm{bp}} / R_{\mathrm{el}}\right)},
$$

where $r$ is the radial distance from the wire, $R_{\mathrm{bp}}$ is the radius of the wire and $R_{\mathrm{el}}=6 \mathrm{~mm}$ is the distance from the wire to the grounded electrode. The potential given by equation (2) results in a deflection, which for small angles is [12]

$$
\delta=\frac{\pi \mathrm{eV} \text { bp }}{2 E_{\mathrm{el}} \ln \left(R_{\mathrm{bp}} / R_{\mathrm{el}}\right)},
$$

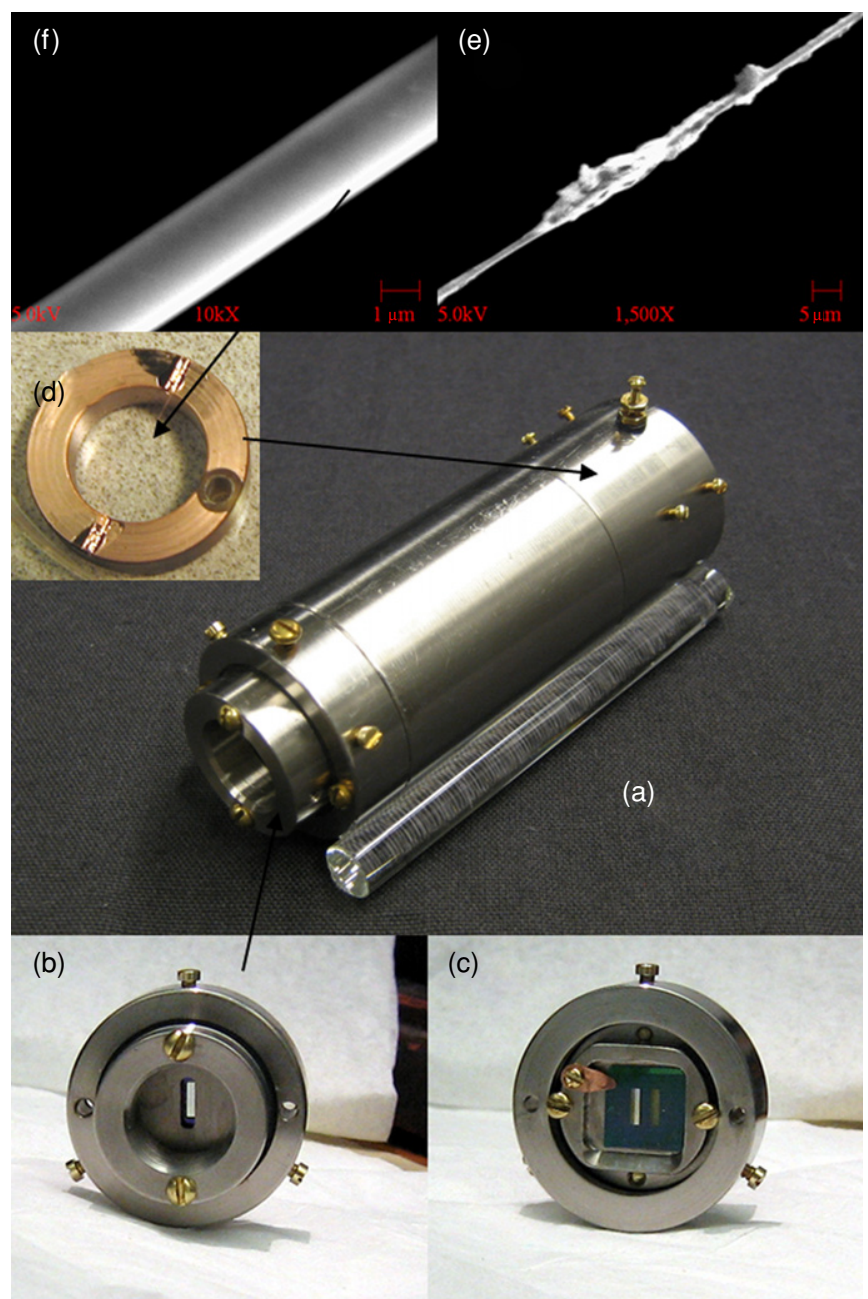

Figure 2. The grating bi-prism electron beam splitter. The titanium $3 \mathrm{~cm}$ diameter mount (a) holds the grating mount (b), (c) and the copper coated Ultem bi-prism mount (d). The front view of the grating mount (b) shows the centred opening that the electrons are incident upon. The back view of the grating mount (c) shows the gold-coated SiN $100 \mathrm{~nm}$ periodicity grating. Care should be taken to select the bi-prism wire. Electron microscope images of a bad-coating run (e) and good-coating run (f) are shown.

where $E_{\mathrm{el}}$ is the kinetic energy of the electron beam expressed in $\mathrm{eV}$. Note that $\delta$ does not explicitly or implicitly depend on $r$, i.e. the deflection angle is independent of the radial distance of the beam from the bi-prism. A negative voltage applied to the wire increases the angle between the first-order beams.

A set of deflection plates is placed $4 \mathrm{~cm}$ downstream from the bi-prism. An electrostatic quadrupole situated $7 \mathrm{~cm}$ behind the bi-prism provides an optional magnification of the diffraction pattern and deflected beams. The detector consists of microchannel plates (MCP) in combination with a phosphor screen, and is located $38 \mathrm{~cm}$ beyond the quadrupole. At $7.5 \mathrm{keV}$, the adjacent diffraction peaks are separated by $75 \mu \mathrm{m}$ (at the detection screen). The peak width is determined by the transverse coherence length [12] and expected to be $10 \mu \mathrm{m}$.

To compare with the experimental results, a quantummechanical numerical simulation was produced. The theoretical description of the physical system is based on Feynman's path integral formulation [14]. Propagation 
from an initial wavefunction given by $\Psi_{i}(x)$ to the final wavefunction $\Psi_{f}(x)$ in the path integral formulation is given by

$$
\Psi_{f}(x)=\int K_{i \rightarrow f}\left(x^{\prime}, x\right) \Psi_{i}\left(x^{\prime}\right) \mathrm{d} x^{\prime} .
$$

The coordinate system is chosen so that the incident electron beam is aligned along the $z$-axis, while the slits and grating are parallel to the $x$-axis. The kernel in equation (4) is given by

$$
K_{i \rightarrow f}\left(x^{\prime}, x\right)=\exp \left(\mathrm{i} S\left(x, x^{\prime}\right) / \hbar\right),
$$

where $S$ is the classical action. For our system, the wavefunction propagates in free space between the planes where the slits, grating, bi-prism and detector are located. For that part of the propagation, the action simplifies to

$$
S\left(x, x^{\prime}\right)=2 \pi l\left(x, x^{\prime}\right) / \lambda_{\mathrm{db}} .
$$

The length of a straight individual Feynman path $l\left(x, x^{\prime}\right)=$ $\sqrt{\left(x-x^{\prime}\right)^{2}+\left(z-z^{\prime}\right)^{2}}$ is measured from some point $\left(x^{\prime}, z^{\prime}\right)$ on a plane to a point $(x, z)$ on a subsequent plane, and $\lambda_{\mathrm{db}}$ is the deBroglie wavelength of the matter wave.

At these planes, the wavefunction is modified in the following way:

$$
\Psi_{\text {plane, out }}(x)=A(x) \exp (\mathrm{i} \varphi(x)) \Psi_{\text {plane, in }}(x) .
$$

For example, at the slit plane the amplitude of the wavefunction is modified by

$$
A_{\text {slit }}(x)=H(x+w / 2) \cdot H(-x+w / 2),
$$

where $w$ is the slit width and $H$ is the Heaviside function, while the phase is unaffected $(\varphi(x)=1)$. For the detailed description of the effect of a grating, see Barwick et al [15]. For the present paper, the description of the bi-prism needs to be added. The bi-prism blocks the electron over its width:

$$
A_{\mathrm{bp}}(x)=H\left(-x-R_{\mathrm{bp}}\right)+H\left(x-R_{\mathrm{bp}}\right) .
$$

The electrons that pass the bi-prism accumulate a phase shift. This phase shift is due to the bi-prism potential given by equation (2). To apply equation (7), the phase shift that is caused by the electron passing through this potential is given by

$$
\varphi_{\mathrm{bp}}(x)=\frac{\mathrm{e}}{\hbar v} \int_{-\infty}^{\infty} V(r(x, z)) \mathrm{d} z
$$

where $v$ is the electron velocity. This integral diverges; however, only local phase differences accumulated for trajectories at different distances from the wire are relevant. Setting the global phase equal to zero at $x=0$ gives

$$
\varphi_{\mathrm{bp}}(x)=\frac{\mathrm{e} \pi}{\hbar v} \frac{V_{\mathrm{bp}}}{\ln \left(R_{\mathrm{bp}} / R_{\mathrm{el}}\right)} x .
$$

Consecutive application of equations (4) and (7) yields the wavefunction at the detection plane from which the probability distribution of the diffraction pattern can be found directly:

$$
P_{\operatorname{det}}(x)=|\Psi(x)|^{2} .
$$

The probability distribution was evaluated numerically, using a 500 processor core cluster at the University of Nebraska's Research Computing Facility.
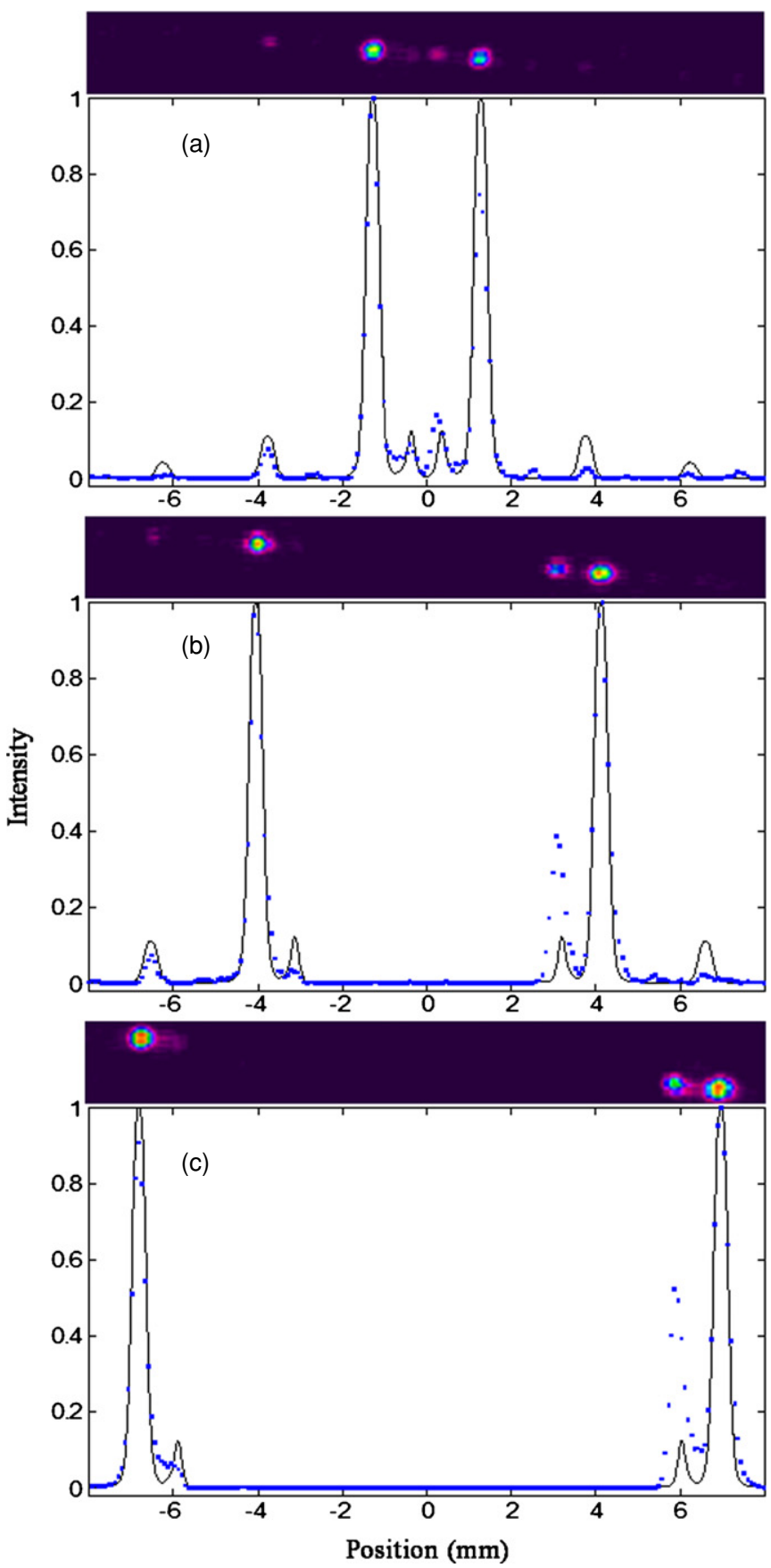

Figure 3. The photographed image of an electron diffraction pattern and the associated line graph are shown for bi-prism voltages of (a) $0 \mathrm{~V}$, (b) $-20 \mathrm{~V}$, (c) $-40 \mathrm{~V}$. Experimental data (blue dots) and a path integral calculation (solid line) are compared. The zero-order diffraction peak is mostly blocked by the bi-prism wire. The diffraction peak separation and width do not substantially change as the bi-prism voltage is increased.

A diffraction pattern with a quadrupole setting producing a magnification of $16 \times$, and zero voltage on the bi-prism, is shown in the graph of figure 3(a). The magnification factor is determined by comparing the measured peak positions to those given by equation (1). The shadow of the bi-prism wire blocks most of the zero-order diffraction peak which is centred around $0 \mathrm{~mm}$ in the graph. The 1st, 3rd, and 5th diffraction orders are visible on the left- and right-hand sides of the 


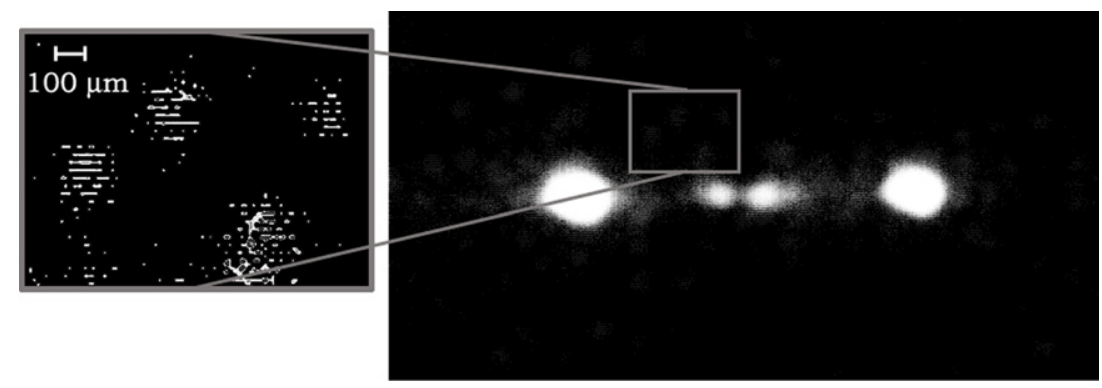

Figure 4. An image of an electron diffraction pattern is shown. In the background, fluorescent spots due to single electrons are visible. The full width at half-maximum (FWHM) of the single-electron spots is in the range 100-150 $\mu \mathrm{m}$, showing that the spatial resolution of the apparatus exceeds the diffraction-order width.

bi-prism shadow. As expected, the even orders are suppressed as a result of using a grating with an open fraction of $50 \%$ [15]. The solid line is the result of the path integral simulation. The simulation result is scaled by a factor to account for the quadrupole magnification. The result of the simulation is fully left-right symmetric, while the data are not. For example, an offset in the bi-prism position can cause the asymmetry in the zero-order remnant. As the voltage on the bi-prism wire is decreased to -20 and $-40 \mathrm{~V}$, the beam separation between the negative and positive diffraction orders increases, while the distance between orders of the same sign does not increase (see figures 3(b) and 3(c)). This indicates, as expected, that the bi-prism deflection angle does not depend on the distance that the electron passes from the bi-prism wire.

To investigate if the grating bi-prism is a useful beam splitter for a large angle electron interferometer, the beam separation needs to be sufficiently large without causing significant beam distortion. Beam distortion can, in general, be described as a position-dependent amplitude and phase variation over the beam profile. Here, the beam distortion is thought to be due to possible phase variations incurred while passing the grating bi-prism combination, while the result is an observable amplitude (probability) variation at the detector plane. A full interferometer would require a second bi-prism wire and/or grating to be installed after the first one to redirect the electron beams towards each other. A separation of $1 \mathrm{~mm}$ (angular beam separation of $2.2 \times 10^{-2}$ radians) between the electron interferometer arms in our device $5 \mathrm{~cm}$ after the bi-prism, at the proposed location of a second biprism, was reached using a bi-prism voltage of $400 \mathrm{~V}$. The observed separation at the detection screen is about $1 \mathrm{~cm}$ with the quadrupole turned off. Thus, the geometric separation at the second bi-prism would be $5 \mathrm{~cm} / L \times 1 \mathrm{~cm}$, where $L$ is the distance between the first bi-prism and the detection screen. Such a separation is a ten-fold increase as compared to any previous electron interferometer design $[1,16]$. To test if there is beam distortion at such large bi-prism voltages, the quadrupole magnification needs to be large enough such that the width of the diffracted beams exceeds the spatial resolution of the detection system.

The spatial resolution of our detection system (including camera) was about $100-150 \mu \mathrm{m}$ as determined from the observed size of individual electron hits (figure 4). The quadrupole increases the beam width to exceed this value.
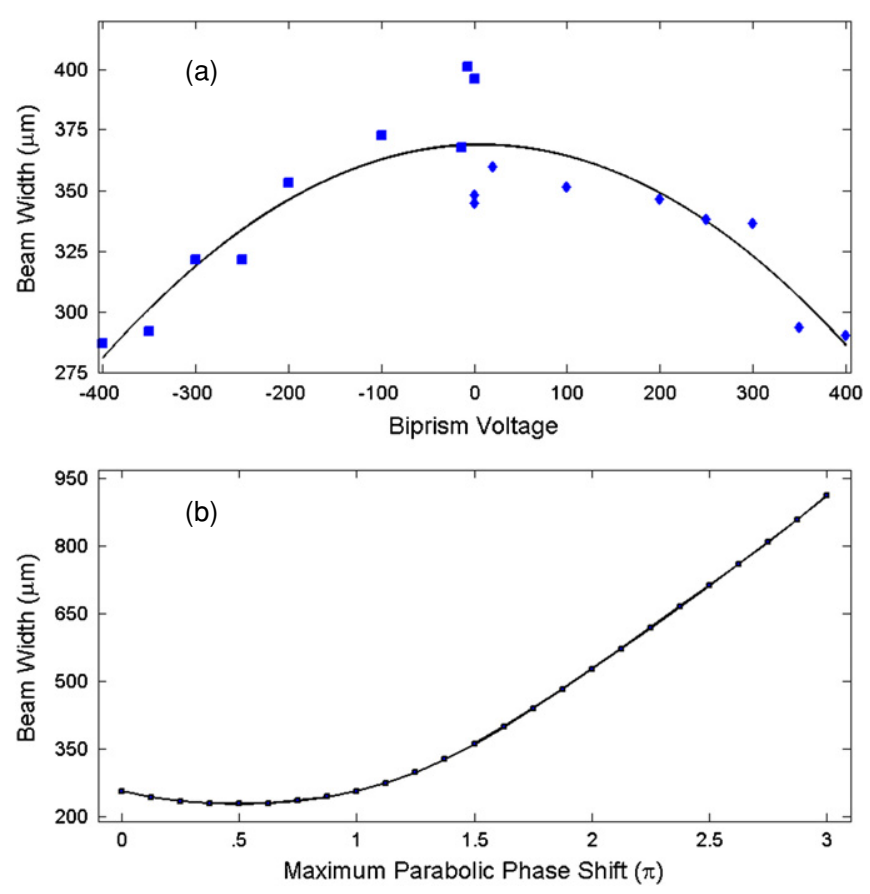

Figure 5. Phase-distortion estimation. (a) The measured diffraction beam width as a function of the bi-prism voltage is shown. The beam narrows by about $100 \mu \mathrm{m}$. (b) A simulation of beam width variation due to a parabolic potential is given. The parabolic potential is applied across the electron beam. The edge of the beam accrues the maximum phase shift. As the potential strength and thus the maximum phase shift are changed, the beam width varies. Starting at a width of about $375 \mu \mathrm{m}$, a width reduction of $100 \mu \mathrm{m}$ requires a phase shift of about $\pi$ radians.

To account for this, the simulation results were first scaled by the magnification factor that the quadrupole provides, and then convoluted with a Gaussian width of $150 \mu \mathrm{m}$. Additionally, moderate electrostatic lensing at the second collimating aperture was added to obtain good agreement between the measured and simulated diffraction peak widths (figures 3 and 5). The lensing was incorporated in the simulation by adding a parabolic phase shift over the width of the second aperture. The width of the observed diffraction orders is about $375 \mu \mathrm{m}$.

In figure 5(a), the measured beam width is shown as a function of applied bi-prism voltage. The major feature is that 
the beam becomes narrower at larger bi-prism voltages. It is important to note that the combined effect of the bi-prism voltage and quadrupole magnification are large enough to shift the electron beam off the detection plate. To overcome this difficulty, the deflection plate in front of the quadrupole was used to keep the position of the beam at the same spot on the detection plate. The beam narrows by about $100 \mu \mathrm{m}$ at bi-prism settings of $\pm 400 \mathrm{~V}$. The same narrowing can be obtained in our simulation by adjusting the lensing strength of the parabolic potential. The maximum phase shift needed to obtain such a narrowing is about $\pi$ radians (figure 5(b)). Phase shifts smaller than this value permit interferometry. It is likely that the phase-shift distortion caused by the bi-prism is much smaller. Reflection symmetry in a plane through the bi-prism wire and parallel to the incident electron beam demands that $V(x)=V(-x)$, where $x$ is orthogonal to the plane. This means that lensing for electrons passing on the left $(x<0)$ or right $(x>0)$ of the wire is the same. For our data, the polarity of the bi-prism voltage is switched for the negative first-order diffraction beam (which passes on the left) as compared to that for the positive first-order diffraction beam (which passes on the right). The lensing, if caused by the bi-prism, should thus be of opposite sign: broadening for negative bi-prism voltages and narrowing for positive bi-prism voltages. This is not observed, and the phase shift distortion is likely due to other electrostatic elements such as the quadrupole.

As the beam-splitting device presented here is a novel combination of previously developed techniques - a material grating and bi-prism-it is useful to compare benefits and drawbacks with other beam-splitting techniques used for electron interferometers. The three-grating Mach-Zehnder interferometer presented in Gronniger et al [5] achieves a maximum beam separation of $3 \mu \mathrm{m}$ (angular separation of $1.2 \times 10^{-4}$ radians) with a grating spacing of $2.5 \mathrm{~cm}$. By comparison, our device can easily attain a distance of $1 \mathrm{~mm}$ (angular separation of $2.2 \times 10^{-2}$ radians) between beams at a distance of $5 \mathrm{~cm}$ behind the bi-prism wire. In order for a three-grating setup to achieve the same separation, the distance between the gratings would need to be approximately $4 \mathrm{~m}$, as there is no beam adjustability present. Moreover, it has been shown that dephasing occurs at the second grating at lower electron energies [5]. The three-grating interferometer loses contrast below energies of $5 \mathrm{keV}$. For a bi-prism interferometer it is known that at energies below $1 \mathrm{keV}$, the interference contrast reduces sharply [17]. The cause of this behaviour is possibly a combination of increased sensitivity to external fields, mechanical alignment details and interaction with nearby surfaces. It has been shown that decoherence can be caused by a purposefully introduced metallic surface near the electron paths in a bi-prism electron interferometer [5]. Bi-prism wires provide metallic surfaces with a close proximity to the electrons. In the operation of bi-prism electron interferometers, great care is used to select a wire with a sufficiently small diameter, a uniform metallic coating, and free of surface contamination.

The idea of a hybrid-grating bi-prism beam-splitterbased interferometer is that the grating will provide some initial distance between the diffracted electron beams and the bi-prism wire to reduce decoherence while keeping the adjustability provided by the potential on the wire to enable a large beam-splitting angle. Hasselbach has shown [9] that contrast reduction by decoherence occurs at an electron-metal surface distance of $d_{\mathrm{m}}=5.5 \mu \mathrm{m}$, and for an interaction time of $\frac{L_{\mathrm{m}}}{v_{\mathrm{m}}}=8.3 \times 10^{-10} \mathrm{~s}$, where $L_{\mathrm{m}}=1 \mathrm{~cm}$ is the electron-surface interaction length. If no grating is used, some electrons will skim the surface of the bi-prism wire and, especially at low electron energies, decoherence may occur. For the grating bi-prism beam splitter, we estimate the electron bi-prism decoherence to be reduced by a factor of $\left(\frac{d_{\mathrm{w}}}{d_{\mathrm{m}}}\right)\left(\frac{d_{\mathrm{w}} / v_{\mathrm{w}}}{L_{\mathrm{m}} / v_{\mathrm{m}}}\right)$ (see [9]), as compared to Hasselbach's experimental situation. For a beam to bi-prism wire distance $d_{\mathrm{w}}=5.5 \mu \mathrm{m}$ and interaction time $d_{\mathrm{w}} / v_{\mathrm{w}}=1.1 \times 10^{-13} \mathrm{~s}$, this factor is about 1000 .

Difficulties in the grating bi-prism approach to interferometry include its sensitivity to mechanical alignment. For example, a slight displacement of the bi-prism wire so that it is not situated in the middle between the two diffraction orders will, upon recombination of the two electron beams, lead to slightly different path lengths. If these exceed the longitudinal coherence, no fringes will be observed. Deflection plates and a Wien filter [17] are needed to overcome these problems, and their installations will be described in forthcoming publications. Mechanical stability of the bi-prism wire in relation to the grating is thus also a concern. A voltage of approximately $6 \mathrm{~V}$ is needed to recombine the positive and negative first-order beams. At that voltage, a wire displacement of about $50 \mathrm{~nm}$ results in a $2 \pi$ phase shift. The wire must thus be vibrationally stable to less than $25 \mathrm{~nm}$ to avoid 'washing out' the interference pattern in time. The previously constructed three-grating Mach-Zehnder interferometer was determined to be stable to $10 \mathrm{~nm}$ or better [5], thus the required stability for the grating bi-prism combination seems achievable. To reach large angles between the beams, the bi-prism voltage is greater and the required mechanical stability increases linearly with the applied voltage. Angular alignment of the grating and wire must also be considered. As a $50 \mathrm{~nm}$ displacement of the wire causes a $2 \pi$ phase shift, the wire displacement at the top of the beam, as compared to the bottom of the beam, must not be shifted by more than $50 \mathrm{~nm}$. At the bi-prism, the beam is approximately $5 \mu \mathrm{m}$ tall; the angular alignment must then be better than $10 \mathrm{mrad}$. Rotational alignment of the gratings to $1 \mathrm{mrad}$ was reached in the three-grating interferometer [5], thus alignment of the grating and bi-prism to $10 \mathrm{mrad}$ is possible.

Electron interferometers utilizing bi-prism filaments have been used extensively in the past 50 years in a wide variety of tasks, and as such are a proven technology. The principal difference of these types of devices from material gratings is that bi-prisms cause wavefront splitting of the electron beam, while gratings are amplitude-splitting devices. Amplitude splitting creates two copies of the incident beam, which are then propagated in space. Wavefront splitting simply divides one wavefront into two, thus the spatial coherence of the original electron wave must exceed the bi-prism wire diameter to allow the two divided wave fronts to interfere when recombined later. Additionally, since the bi-prism is placed 
directly in the path of the wavefront, surface effects due to the wire are more pronounced than in our device where the beams are spatially separated from the bi-prism. Furthermore, the largest beam separation bi-prism interferometers obtained is about $120 \mu \mathrm{m}[1,16]$. The question of how large a beam separation in an interferometer can be achieved using material gratings is an open one. However, a grating bi-prism combination seems more suited to explore this than the use of multiple gratings given its ability to produce relatively large separation distances in a small apparatus size, as discussed above. We have demonstrated a separation of $1 \mathrm{~mm}$ at a distance of $5 \mathrm{~cm}$ after the bi-prism location with little distortion.

\section{Acknowledgments}

This material is based upon work supported by the National Science Foundation under grant no. 0653182. This work was completed utilizing the Research Computing Facility with the associated USCMS Tier-2 site at the University of NebraskaLincoln.

\section{References}

[1] Tonomura A 1999 Electron Holography (Springer Series in Optical Sciences, vol 70) 2nd edn (Berlin: Springer) p 162

[2] Marton L, Simpson J A and Suddeth J A 1953 Electron beam interferometer Phys. Rev. 90490

[3] Marton L, Simpson J A and Suddeth J A 1954 An electron interferometer Rev. Sci. Instrum. 251099

[4] Möllenstedt G and Düker H 1955 FRESNELscher interferenzversuch mit einem biprisma für elektronenwellen Naturwissenschaften 4241
[5] Gronniger G, Barwick B and Batelaan H 2006 A three grating electron interferometer New J. Phys. 8224

[6] Cronin A and McMorran B 2006 Electron interferometry with nanogratings Phys. Rev. A 74061602

[7] McMorran B J and Cronin A D 2009 An electron Talbot interferometer New J. Phys. 11033021

[8] Forrey R C, Dalgarno A and Schmiedmayer J 1999 Determining the electron forward-scattering amplitude using electron interferometry Phys. Rev. A 59942

[9] Sonnentag P and Hasselbach F 2007 Measurement of decoherence of electron waves and visualization of the quantum-classical transition Phys. Rev. Lett. 98200402

[10] Ozcan M 1998 Influence of electric potentials on atom interferometers: increased rotation sensitivity J. Appl. Phys. 83 6185-6

[11] Badurek G, Weinfurter H, Gahler R, Kollmar A, Wehinger S and Zeilinger A 1993 Nondispersive phase of the Aharonov-Bohm effect Phys. Rev. Lett. 71307

[12] Gronniger G, Barwick B, Batelaan H, Savas T, Pritchard D and Cronin A 2005 Electron diffraction from free-standing, metal-coated transmission gratings Appl. Phys. Lett. 87124104

[13] Hibi T and Yada K 1976 Electron interference microscopy Principles and Techniques of Electron Microscopy ed M A Hayat (New York: Van Nostrand-Reinhold) pp 312-43

[14] Feynman R P and Hibbs A R 1965 Quantum Mechanics and Path Integrals (New York: McGraw-Hill))

[15] Barwick B, Gronniger G, Yuan L, Liou S and Batelaan H 2006 A measurement of electron-wall interactions using transmission diffraction from nano-fabricated gratings J. Appl. Phys. 100074322

[16] Möllenstedt G and Bayh W 1962 Kontinuierliche Phasenschiebung von elektronenwellen im kraftfeldfreien raum durch das magnetische vektorpotential eines solenoids Phys. Blätt. 18299

[17] Nicklaus M and Hasselbach F 1993 Wien filter: a wavepacket-shifting device for restoring longitudinal coherence in charged-matter-wave interferometers Phys. Rev. A 48152 